\title{
Topographic instability of flow in a rotating fluid
}

\author{
K. I. Patarashvili ${ }^{1}$, Z. J. Tsakadze ${ }^{1,2}$, M. V. Kalashnik ${ }^{3}$, V. O. Kakhiani ${ }^{2}$, R. J. Chanishvili ${ }^{2}$, J. I. Nanobashvili ${ }^{2}$, and \\ M. A. Zhvania ${ }^{2}$ \\ ${ }^{1}$ E. Andronikashvili Institute of Physics, 6 Tamarashvili str.,Tbilisi 0171, Georgia \\ ${ }^{2}$ Abastumani Astrophysical Observatory, 2a Kazbegi ave., Tbilisi 0161, Georgia \\ ${ }^{3}$ Obninsk State Technical University of Atomic Energy (IATE), 1 Studgorodok, Kaluga Regiont, Obninsk 249040, Russia
}

Received: 16 January 2006 - Revised: 24 April 2006 - Accepted: 26 April 2006 - Published: 21 June 2006

\begin{abstract}
Here are presented the results of experimental and theoretical studies on a stability of zonal geostrophic flows in the rotating layer of the shallow water. In the experiments, a special apparatus by Abastumani Astrophysical Observatory Georgian Academy of Science was used. This apparatus represents a paraboloid of rotation, which can be set in a regulable rotation around the vertical axis. Maximal diameter of the paraboloid is $1.2 \mathrm{~m}$, radius of curvature in the pole is $0.698 \mathrm{~m}$. In the paraboloid, water spreads on walls as a layer uniform on height under the period of rotation $1.677 \mathrm{~s}$. Against a background of the rotating fluid, the zonal flows are formed by the source-sink system. It consists of two concentric circular perforations on the paraboloid bottom (width is $0.3 \mathrm{~cm}$, radiuses are 8.4 and $57.3 \mathrm{~cm}$, respectively); water can be pumped through them with various velocities and in all directions. It has been established that under constant vertical depth of the rotating fluid the zonal flows are stable. There are given the measurements of the radial profiles for the water level and velocity in the stationary regime. It has been found that zonal flows may lose stability under the presence of the radial gradient of full depth formed by a change of angular velocity of paraboloid rotation. An instability origin results in the loss of flow axial symmetry and in the appearance of self-excited oscillations in the zonal flow. At the given angular velocity of rotation, instability is observed only in the definite range of intensities of the source-sink system.
\end{abstract}

The theoretical estimations are performed in the framework of the equations of the shallow water theory, including the terms describing the bottom friction. It has been shown that the instability of zonal flows found experimentally has a topographical nature and is related with non-monotone dependence of the potential vorticity on radius.

\footnotetext{
Correspondence to: M. V. Kalashnik

(lingel@obninsk.com)
}

\section{Introduction}

In the present report we consider a mechanism of topographic instability of a flow in a rotating fluid. In contrast to traditional shear induced instabilities, this type of instability is associated with non-uniform distribution of depth of the fluid with radius. Topographic instability is revealed in experiments with water in a paraboloid rotating around the vertical axis. The fluid in such a rotating basin is distributed as a thin layer along the surface of the basin and has important features in common with planet atmospheres (Nezlin and Snejkin, 1990). Zonal flows in the paraboloid are induced by a source-sink system of mass. A radial flow that arises between concentric source and sink of mass is transformed into an azimuthal (zonal) flow under the Coriolis force action.

\section{Theoretical consideration}

Consider the paraboloid filled with fluid, with profile of the bottom $z=D(r)=\kappa r^{2} / 2$, and rotating with an angular velocity $\Omega$ about the upward-directed $z$-axis. It is easy to show that in the absence of flows the total depth of the fluid in the paraboloid measured along the vertical direction from the bottom is

$h(r)=H-h_{1}(r) \equiv H-\left[1-\left(\Omega / \Omega_{*}\right)^{2}\right] \kappa r^{2} / 2$,

where $H=$ const is the standard depth (at $r=0$ ), $\Omega_{*}=\sqrt{g \kappa}$ is the standard angular velocity, and $g$ is the gravitational acceleration. If $\Omega=\Omega_{*}$, then $h(r)=H=$ const; for $\Omega \neq \Omega_{*}$, we have the non-zero gradient of the total depth $d h / d r=\left[\left(\Omega / \Omega_{*}\right)^{2}-1\right] \kappa r$. Suppose now that in the rotating paraboloid a flow with the azimuthal velocity field $\mathrm{v}(r)$ is created. In the rotating frame of reference the deviation $\eta(r)$ of the total depth from Eq. (1) is associated with this

Published by Copernicus GmbH on behalf of the European Geosciences Union and the American Geophysical Union. 
flow. For small velocities the deviation can be found from the geostrophic relation (Doljanskii et al., 1990)

$\mathrm{v}=\frac{g}{f} \frac{\partial \eta}{\partial r}$,

$f=2 \Omega$ is the Coriolis parameter. Thus, in the presence of the flow the total depth of the fluid in the paraboloid is $h(r)=H-h_{1}(r)+\eta(r)$.

Consider now a flow produced by a given distribution $Q(r)$ of the sources and sinks in the paraboloid. We take a distribution $Q(r)$ in the following form:

$Q(r)= \pm Q_{0}\left[\frac{\delta\left(r-r_{2}\right)}{2 \pi r}-\frac{\delta\left(r-r_{1}\right)}{2 \pi r}\right]$,

where $\delta(r)$ is delta-function, and consider the flow in the interval $r_{1} \leq r \leq r_{2}$. Such a distribution corresponds to concentrated source and sink with an equal intensity $Q_{0}$ (it defines the total volume of the fluid that runs in/out per unit time). If we choose the positive sign in Eq. (3), then source is situated at a radius $r_{2}$, otherwise - at $r_{1}$.

The structure of velocity field in the paraboloid for the source-sink distributions (Eq. 3) was studied by Danilov and Sazonov (1999). There was used the shallow water model with a bottom friction. If the source is located on the radius $r_{2}$, then azimuthal velocity is

$\mathrm{v}(r)=\frac{f}{2 p r}\left[1-\exp \left(p\left(r^{2}-r_{2}^{2}\right)\right)\right], \quad p=\frac{\pi \lambda}{Q_{0}}$

where $\lambda=v / \delta_{E}$ is the coefficient of boundary friction, $\delta_{E}=\sqrt{v / \Omega}$ is the thickness of the Ekman boundary layer, $v$ is the kinematic viscosity of the fluid. If the source is located on the radius $r_{1}$,

$\mathrm{v}(r)=\frac{f}{2 p r}\left[\exp \left(p\left(r_{1}^{2}-r^{2}\right)\right)-1\right]$.

For sufficiently weak intensities $Q_{0} \rightarrow 0 \quad(p \rightarrow \infty)$, from Eqs. (4), (5) the asymptotic expressions follow:

$\mathrm{v}(r)= \pm \frac{Q_{0}}{\pi \delta_{E}} \frac{1}{r}$.

By using Eq. (6) and geostrophic relation, for the total deviation of the depth we have

$\eta(r)= \pm\left(C+\frac{f Q_{0}}{\pi g \delta_{E}} \ln r\right)$.

Here a constant $C$ is found from the condition $\int_{r_{1}}^{r_{2}} \eta r d r=0$ (mass conservation).

Investigate the stability of axially symmetric flow $\mathrm{v}(r)$ in the non-viscous limit.

The important feature of this flow is the potential vorticity

$q(r)=\frac{\omega(r)+f}{h(r)}, \quad \omega(r)=\frac{1}{r} \frac{d}{d r}(r v)$.
Considering the linearized form of the shallow water equations, one can show easily that a necessary condition for instability of the flow is the following: the radial gradient of the potential vorticity $d q / d r$ vanishes somewhere in the field of a flow (Pedlosky, 1982; Dikii, 1976). In the quasigeostrophic theory of the shallow water this condition is usually called the Kuo's criterion or the Charney-Kuo theorem. It is a straightforward generalization of the well-known Rayleigh's inflection-point theorem.

Let us apply the above mentioned criterion to the flow (Eq. 6). Particularly, we consider the case of cyclonic rotation (i.e. the positive sign in Eq. 6). Since for the flow (Eq. 6) the vertical component of the vorticity $\omega=r^{-1} d(r \mathrm{v}) / d r \equiv 0$, the radial gradient of $q$ is connected only to the change of the total depth:

$\frac{d q}{d r}=-\frac{f}{h^{2}} \frac{d}{d r}\left(-h_{1}+\eta\right)=\frac{f}{h^{2}}\left\{\left[1-\left(\Omega / \Omega_{*}\right)^{2}\right] \kappa r-\frac{f Q_{0}}{\pi g \delta_{E}} \frac{1}{r}\right\}$.

It follows from Eq. (9) that $d q / d r$ can vanish at some point only if $\Omega<\Omega_{*}$, i.e., for angular velocities smaller than the standard angular velocity. In this case for the point $r_{*}$, where $d q / d r=0$, we have

$r_{*}^{2}=\frac{f Q_{0}}{\pi \delta_{E}\left(\Omega_{*}^{2}-\Omega^{2}\right)}$.

According to the instability criterion, this point should be within the interval $r_{1} \leq r \leq r_{2}$, in other words, the condition $r_{1}^{2} \leq r_{*}^{2} \leq r_{2}^{2}$ should be satisfied. Taking into account Eq. (10), the latter criterion can be rewritten as a constraint on the intensity $Q_{0}$ :

$Q_{1} \leq Q_{0} \leq Q_{2}$

with the threshold values

$Q_{i}=\frac{\pi \delta_{E} r_{i}^{2}\left(\Omega_{*}^{2}-\Omega^{2}\right)}{2 \Omega}, \quad i=1,2$.

From Eq. (1) it follows that for a fixed angular velocity satisfying the condition $\Omega<\Omega_{*}$ there exists a range of $Q_{0}$ where instability may be observed. Increasing gradually the values of $Q_{0}$ from zero, in the beginning we are in the unstable region and then, for $Q_{0}>Q_{2}$, we leave this region and move to the stable region.

The case of anticyclonic flows (when there is minus in Eq. 6) can be analyzed in a similar manner. A necessary condition for non-viscous instability is now met only if $\Omega>\Omega_{*}$ in a certain interval of $Q_{0}$.

Let's emphasize that analysis has a qualitative character. The rigorous proof of instability may be derived only from the solution of corresponding spectral problem on the theory of stability. Formulating this problem should use the accurate expressions for velocity (4), (5), as well as take into account the spiral character of the flow related to the presence of the radial stream. 


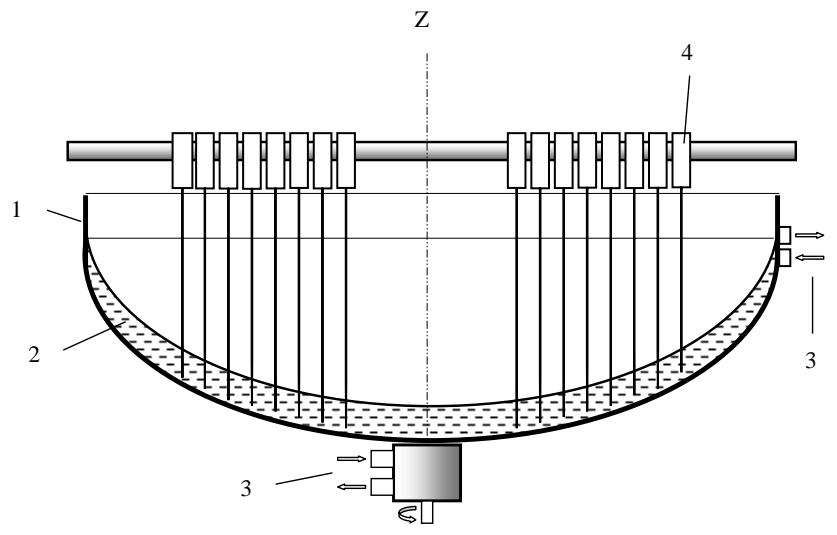

Fig. 1. The cartoon of a mechanical part of experimental set-up: 1 - rotating container with a parabolic bottom, 2 - layer of a fluid, 3 - system of radial circulation of a fluid, 4 - capacitance transducers of fluid thickness.

\section{Experiment}

The experimental investigation of the instability was carried out in the Abastumani Astrophysical Observatory on the special device - the paraboloid of rotation with the maximum diameter $d=1.2 \mathrm{~m}$ and curvature radius in a pole $R=1 / \kappa=0.698 \mathrm{~m}$ (Fig. 1). For this paraboloid, the standard angular velocity is $\Omega_{*}=\sqrt{g \kappa}=3.75 \mathrm{~s}^{-1}$, accordingly, the standard period of one revolution is $T_{*}=2 \pi / \Omega_{*}=1.677 \mathrm{~s}$. The azimuthal (zonal) flow in the paraboloid was produced by a source-sink system of mass consisting of two concentric circular slots $0.3 \mathrm{~cm}$ in width, situated at the bottom of the paraboloid at the radii $r_{1}=8.4 \mathrm{~cm}$ and $r_{2}=57.3 \mathrm{~cm}$. Through these slots the working fluid (water) was pushed with the given total rate $Q_{0}\left(\mathrm{~cm}^{3} / \mathrm{s}\right)$ and in a given direction. The detailed description of experimental device and measurement instrumentation are given in the work by Zhvania et al. (2006).

A series of experiments were performed with various values of $Q_{0}$, the depth $H$ in the absence of fluid motion, and the angular velocity of the paraboloid $\Omega$. During the experiments, the depth of the fluid was measured by means of variable-capacitance transducers placed along the radius of the basin. For the measurements of the velocity, a method of tracer particles was used. The main results of our experiments are:

1. When the paraboloid rotates with the angular velocity $\Omega=\Omega_{*}$, the azimuthal flows induced by a source-sink system of mass are stable.

2. In the case of cyclonic flows (a source is at the radius $r_{2}$ ), the instability of azimuthal flows is observed only for angular velocities that satisfies the condition $\Omega<\Omega_{*}$; for anticyclonic flows the contrary holds $\left(\Omega>\Omega_{*}\right)$.

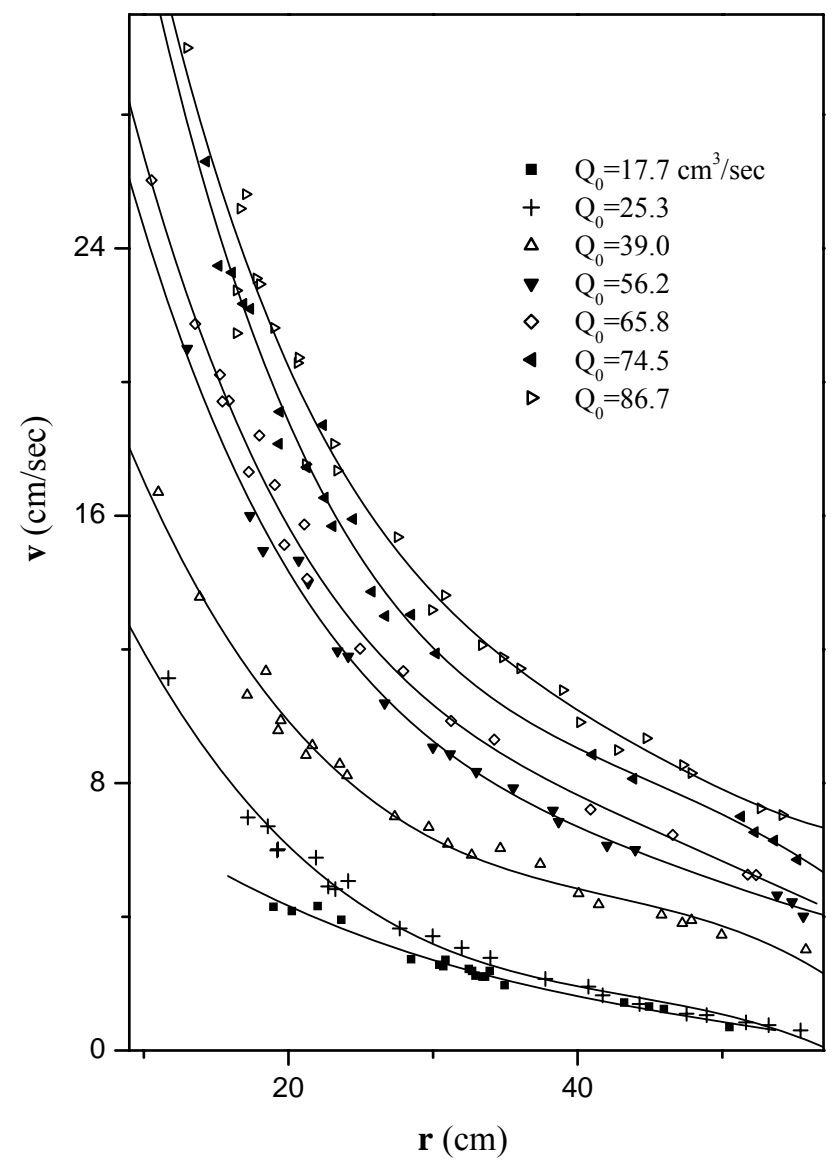

Fig. 2. Experimental profiles of $\mathrm{v}(r)$ for various values of the intensity $Q_{0}$ and angular velocity $\Omega=\Omega_{*}$. A source of mass is situated at a radius $r_{2}$.

3. For a fixed angular velocity $\Omega$, the instability exists only in a certain range of values of the intensity $Q_{0}$.

4. The instability leads to the breaking of axial symmetry of the flow and to auto-oscillations occurrence in the flow. The structure of a non-axially symmetric flow is dominated by the mode with the azimuthal wave number $m=2$. The period of arisen auto-oscillations is several times greater (practically an order of magnitude) than the period of rotation of the system.

In Fig. 2 we plot the experimental profiles of $\mathrm{v}(r)$ obtained for various values of $Q_{0}$ and the angular velocity $\Omega=\Omega_{*}$ in the case of cyclonic flow. The characteristic time of the statement of profiles, after switching on the source-sink system, is 1-2 min. These experimental profiles do not show any signs of instability and are in good agreement with the theoretical ones (Eq. 6).

In Fig. 3a we present the dependence of the total depth on time at various radii obtained for the angular velocities $\Omega<\Omega_{*}$ (cyclonic case). This figure demonstrates the process of formation of the instability leading to auto-oscillations 

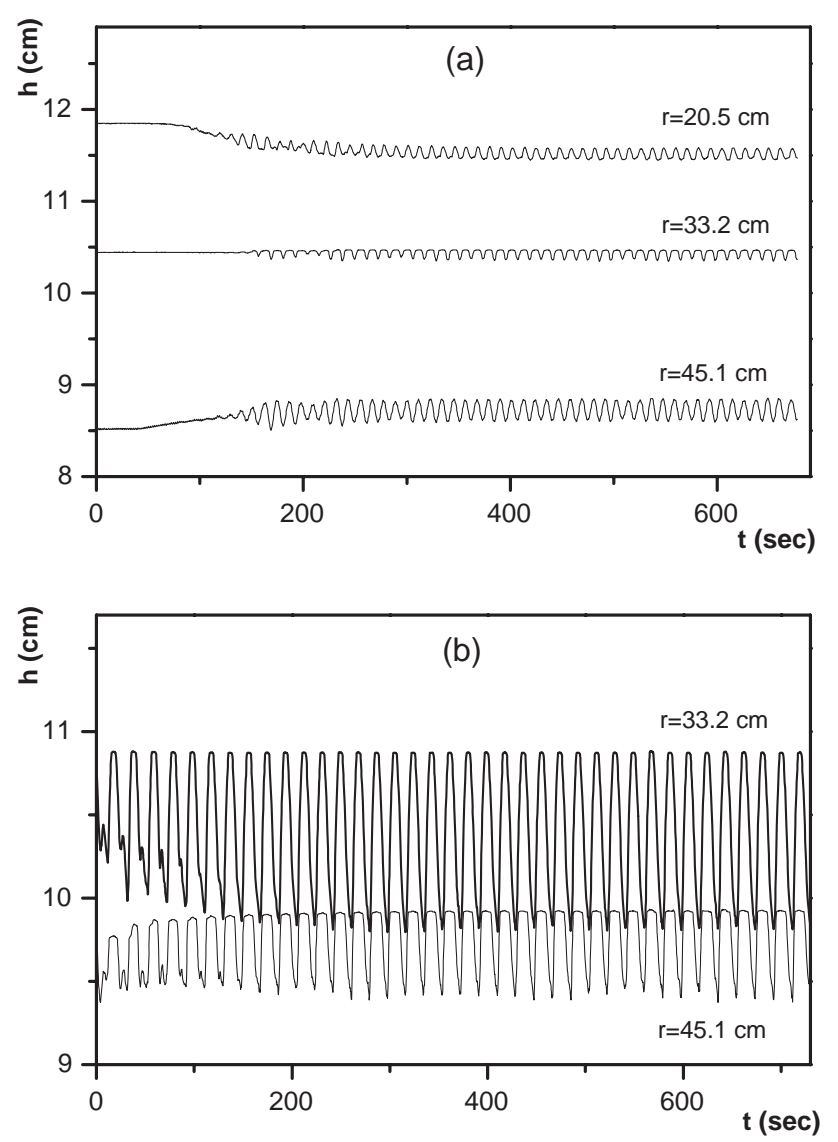

Fig. 3. Dependence of the total depth on time at various radii for angular velocities $\Omega<\Omega_{*}$.

when the value of $Q_{0}$ grows from 0 to $25 \mathrm{~cm}^{3} / \mathrm{s}$. The structure of oscillations of the total depth in the regime of fully developed instability $\left(Q_{0}=90 \mathrm{~cm}^{3} / \mathrm{s}\right)$ is shown in Fig. 3b. Notice the distinctively non-harmonic nature of oscillations that is, perhaps, associated with their nonlinearity. To find the characteristic period of oscillations, we calculated the corresponding time spectra. For $Q_{0}=104 \mathrm{~cm}^{3} / \mathrm{s}$ and the angular velocity $\Omega=3.095 \mathrm{~s}^{-1}$ (the period of rotation is $T=2.03 s>T_{*}$ ), the chief maximum in the spectrum corresponds to the period $T_{W}=16.9 \mathrm{~s}$ of oscillations of the fluid. This value is more than eight times larger than the period of rotation of the system. The data from experiments carried out for various values of the intensity showed that the period $T_{W}$ decreases with increasing $Q_{0}$ and this dependence is almost linear.

Figure $4 \mathrm{a}$ is quite instructive. It shows how instability decays (oscillations damp) when we further increase the intensity from $Q_{0}=104 \mathrm{~cm}^{3} / \mathrm{s}$ to $Q_{0}=140 \mathrm{~cm}^{3} / \mathrm{s}$. Figure $4 \mathrm{~b}$ illustrates the opposite process - the emergence of oscillations when the intensity decreases from $Q_{0}=160 \mathrm{~cm}^{3} / \mathrm{s}$ to $Q_{0}=55 \mathrm{~cm}^{3} / \mathrm{s}$ (return to the unstable region). The figures presented here clearly confirm the existence of two threshold
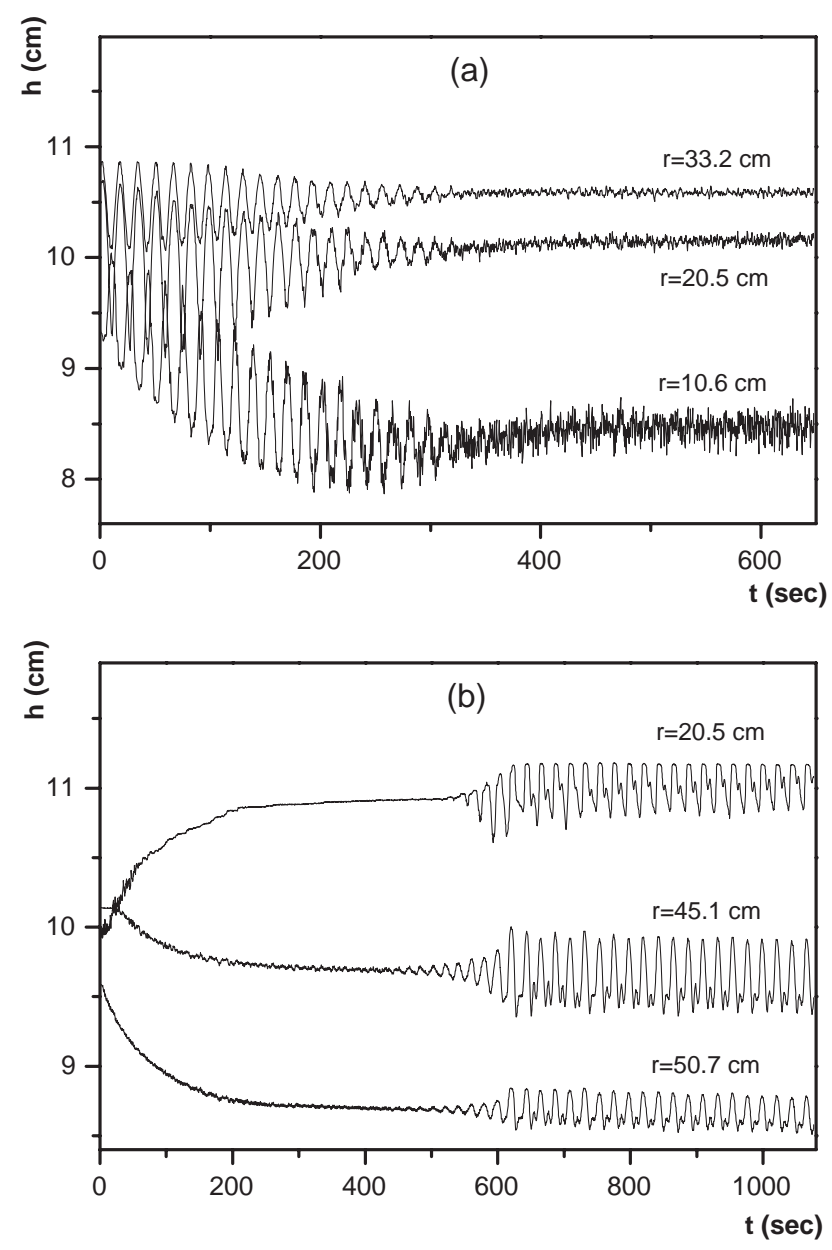

Fig. 4. Dependence of the total depth on time at various radii.

values $Q_{1}, Q_{2}$ determining the development of instability in the range $Q_{1}<Q_{0}<Q_{2}$. Let us evaluate this values for an experiment in which the angular velocity $\Omega=3.095 \mathrm{~s}^{-1}$. For the parameters of the paraboloid given above and kinematic viscosity of water $\nu=10^{-2} \mathrm{~cm}^{2} / \mathrm{s}$, calculations from Eq. (12) derive that $Q_{1}=9.12 \mathrm{~cm}^{3} / \mathrm{s}, Q_{2}=427 \mathrm{~cm}^{3} / s$. It is obvious that the value of $Q_{2}$ almost three times exceeds the actual one. This discrepancy is quite understandable: firstly, for large intensities instead of Eq. (6) we should use more accurate expressions for the flow velocity, and secondly, when deriving Eq. (11) we have used the necessary criterion for non-viscous instability and, in fact, we can say that instability takes place only for the certain values of $Q_{0}$ from the range $\left(Q_{1}, Q_{2}\right)$.

\section{Conclusions}

In conclusion, we would like to stress that the instability investigated in this paper differs fundamentally from that studied in the known works Nezlin and Snejkin (1990), as well 
as Doljanski et al. (1990). In these papers a common shear instability was modeled (modified by rotation), provoked by the flows directed oppositely. By contrast, in the above studied instability, it is the gradient of the total depth, not shear, that plays a major role, i.e., this instability is of topographic origin. The fact that this instability can be so easily produced in laboratory experiments, makes it very attractive for further theoretical and experimental explorations.

Acknowledgements. The authors would like to thank L. K. Ingel for discussions and G. R. Mamatsashvili for the translation of the paper. This work was supported by the International Science and Technology Center grant G-1217.

Edited by: N. S. Erokhin

Reviewed by: V. Ponomarev and two other referees

\section{References}

Danilov, S. D. and Sazonov, I. A.: Calculation of a zonal sourcesink flow in a rotating basin, Izv. Acad. Sci. Russia, Atmos. Ocean. Phys., 35, 344-355, 1999.

Dikii, L. A.: Hydrodynamic stability and dynamics of the atmosphere, Gidrometeoizdat, Leningrad, 1976.

Doljanski, F. V., Krimov, V. A., and Manin, D. U.: A stability and vortex structure of quasi-dimensional shear flows, Sov.Phys. Uspekhi, 160, 1-47, 1990.

Nezlin, M. V. and Snejkin, E. N.: Rossby vortices and spiral structure, Nauka, Moscow, 1990.

Pedlosky, J.: Geophysical fluid dynamics, Springer-Verlag, BerlinHeidelberg-New-York, 1982.

Zhvania, M. A., Kalashnik, M. V., Kakhiani, V. O., Nanobashvili, J. I., Patarashvili, K. I., and Tsakadze, Z. J.: Formation of azimuthal flows created by the source-sink system in the rotating paraboloid, Izvestia RAN. Fluid Mechanics, 2, 60-74, 2006. 